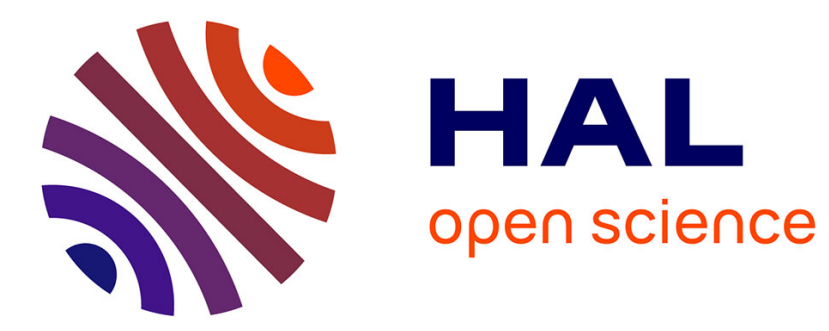

\title{
Coherent signal from incoherently cw-pumped singly resonant Ti:LiNbO3 integrated optical parametric oscillators
}

Carlos Montes, Werner Grundkötter, Hubertus Suche, Wolfgang Sohler

\section{- To cite this version:}

Carlos Montes, Werner Grundkötter, Hubertus Suche, Wolfgang Sohler. Coherent signal from incoherently cw-pumped singly resonant Ti:LiNbO3 integrated optical parametric oscillators. Journal of the Optical Society of America B, 2007, 24 (11), pp.2796-2806. hal-00433404

\author{
HAL Id: hal-00433404 \\ https://hal.science/hal-00433404
}

Submitted on 25 Feb 2010

HAL is a multi-disciplinary open access archive for the deposit and dissemination of scientific research documents, whether they are published or not. The documents may come from teaching and research institutions in France or abroad, or from public or private research centers.
L'archive ouverte pluridisciplinaire HAL, est destinée au dépôt et à la diffusion de documents scientifiques de niveau recherche, publiés ou non, émanant des établissements d'enseignement et de recherche français ou étrangers, des laboratoires publics ou privés. 


\title{
Coherent signal from incoherently cw-pumped singly resonant $\mathrm{Ti}: \mathrm{LiNbO}_{3}$ integrated optical parametric oscillators
}

\author{
Carlos Montes \\ Centre National de la Recherche Scientifique \\ Laboratoire de Physique de la Matière Condensée \\ Université de Nice - Sophia Antipolis, Parc Valrose, \\ F-06108 Nice Cedex 2, France \\ and
}

Werner Grundkötter, Hubertus Suche, and Wolfgang Sohler Angewandte Physik, Universität Paderborn

Warburger Strasse 100, D-33098 Paderborn, Germany

Corresponding author: Carlos.Montes@unice.fr

Received May 10, 2007; revised August 22, 2007; accepted August 31, 2007; posted September 10, 2007 (Doc. ID 82856); published October 12, 2007 in J. Opt. Soc. Am. B/Vol. 24, No. 11/November 2007 


\begin{abstract}
Incoherent broad-bandwidth cw-pumping singly resonant Ti:LiNbO 3 integrated optical parametric oscillators, in the quasi-phase-matched nondegenerate type I $\{e e e\}$ configuration, may efficiently generate coherent signal output by the convectioninduced phase-locking mechanism. The incoherence of the pump is absorbed by the idler wave, propagating at the same group velocity, while the finite temporal walk-off of the signal with respect to the pump and idler waves determines a signal coherence gain of more than 4 orders of magnitude. (c)2007 Optical Society of America.
\end{abstract}

OCIS codes: 030.1640, 190.4970, 130.4310,

Keywords: Coherence; Parametric oscillators; Nonlinear integrated optics 


\section{Introduction}

Quasi-phase-matched optical parametric oscillators (OPO) are low-power compact sources of tunable coherent near- and mid-infrared wavelength radiation offering very efficient frequency conversion, continuously tunable in a broad wavelength range [1]. Singly resonant cw-pumped OPOs (SOPOs), with feedback only at the signal wavelength, offering simplified tuning and strongly enhanced stability have been reported in nonlinear $\chi^{(2)}$ crystals such as potassium phophate (KTP) $[2,3]$ and lithium niobate (LN) with direct laser diode pumping [4]. Waveguide geometries considerably increase the conversion efficiency of nonlinear devices by tightly confining the optical fields over long interaction lengths [5] - [7]. More specifically, singly resonant integrated OPOs or SR-IOPOs, now available as low-threshold cw-pumped integrated devices [6] - [10], constitute diffractionless configurations very attractive for the demonstration of the mechanism we propose in this paper. Usually, they are pumped by narrow linewidth sources as the spectral properties of the pump are transferred to the signal and idler. However, this coherence transfer is strongly determined by the dispersion properties of the waveguide material leading to surprising phenomena. We investigate here the coherence properties of the parametric nondegenerate three-wave interaction driven from an incoherent pump and we look for the coherence transfer from a broad-bandwidth and high fluctuating cw pump to a narrow-bandwidth and lowly fluctuating signal. For this purpose, we take advantage of the convection-induced phase-locking mechanism discussed in previous studies [?], based on a group velocity difference between the resonant signal wave and the pump and idler waves (or temporal walk-off), and a good group velocity mismatch between the pump and the idler waves. However, in contrast with the study proposed in [13] for two experimental configurations of type II singly resonant IOPOs, we show here the feasibility of coherent signal generation from an incoherent cw pump in a type I $\{e e e\}$ singly resonant Ti:LiNbO 3 IOPO working at $\lambda_{s}=3.94 \mu \mathrm{m}$, near the infrared transparency limit. The main reason is to benefit for the highest nonlinear $\chi^{(2)}$ coefficient in a more simple configuration, while preserving a sufficiently large group velocity difference between the signal wave and the pump and idler waves, namely, $\Delta v / v_{p} \equiv\left|v_{s}-v_{p}\right| / v_{p} \simeq 1 / 44$, which is necessary to en- 
sure the phase-locking mechanism. Moreover, it seems possible to prepare an incoherent pump at $\lambda_{p}=1.535 \mu \mathrm{m}$, like an amplified spontaneous emission (ASE) source amplified in an Er-doped fiber, instead of the less broader bandwidth diode sources commonly used to pump periodically poled lithium niobate (PPLN) bulk singly-resonant cw OPOs [4]. However, to determine the choice of these resonant wavelength-sets, we have performed many simulations with a large set of different pump power, cavity length and cavity finesse parameters.

The three-wave parametric interaction in a quasi-phase matched OPO consists in an amplification process where energy is transferred from the pump wave $A_{p}$ to the daughter waves, called signal $A_{s}$ and idler $A_{i}$. It is common to call idler the wave of the largest wavelength, since the OPOs are habitually resonant at the intermediate wavelength. Here, the singly resonant wave is at the largest wavelength and we will call it signal. If the cw pump is generated by a single mode laser source with a broad-bandwidth random phase spectrum, the three-wave interaction generally transfers this incoherence to the daughter waves, as described by the usual kinetic approach based on the random phase approximation (RPA) [14] - [18]. In contrast to this usual approach, we have shown that the convection between the interacting waves is responsible for a phase-locking mechanism which leads to the generation of a coherent signal, despite of the incoherence of the pump. The physics of this three-wave mechanism is well described in Refs. [11, 12], where a coherent localized (soliton) signal was shown to be generated and sustained by an incoherent source provided that, (i) the group velocities of the pump and idler waves are almost matched $\left(v_{p} \simeq v_{i}\right)$, and (ii) the group velocity difference between the pump and signal waves is large enough. To briefly recall the essential properties of the phaselocking mechanism, let us note that this mechanism is only significant when the parametric interaction takes place in the incoherent regime of interaction defined by the inequality

$$
\tau_{c}^{e f f}=\left(v_{p} / \Delta v\right) \tau_{c}<\tau_{0}
$$

where $\Delta v=\left|v_{p}-v_{s}\right|$ refers to the group velocity difference between the pump and signal waves (or temporal walk-off), $\tau_{c}$ refers to the correlation time of the incoherent pump wave, 
and $\tau_{0}$ refers to the nonlinear characteristic time of the parametric three-wave interaction (or growth time), which is the inverse of the product of the pump input amplitude $E_{p}(0)$ times the nonlinear coupling coefficient $\sigma_{p} / 2\left[\tau_{0}=2 /\left[E_{p}(0) \sigma_{p}\right]\right.$, cf. Sections 3. and 4.]. Therefore $\tau_{c}^{\text {eff }}$ represents the effective time correlation of the pump wave as it is seen by the signal due to its convection with respect to the pump. The inequality (1) then simply expresses the fact that we are considering a genuine incoherent regime of interaction, in which the random-phase approximation is usually applied. In this regime, the signal wave is expected to follow the rapid phase-fluctuations of the pump wave, and would not be able to reach a coherent state. However, in contrast with this common belief, it was shown in Refs.[11, 12] that if the pump and idler group velocities are almost matched, the idler field may absorb the incoherence of the pump, thus allowing the signal to evolve towards a highly coherent state. Let us underline that such a pump-idler phase-locking mechanism does not require an exact matching of the corresponding velocities, it is indeed sufficient that the velocities obey the following criterion $[11,12]$

$$
\left|v_{p}-v_{i}\right|<\tau_{c} v_{p} \gamma_{i}
$$

to allow the idler phase to follow the rapid pump phase fluctuations.

[We give the mathematical arguments sustaining the phase-locking mechanism in Appendix C, once the dynamical equations have been introduced.]

The coherence properties of the generated signal require the evaluation of the autocorrelation function. It is shown in [12] to be essentially governed by the rate $r=\tau_{c}^{e f f} / \tau_{0}$ entering inequality (1). For large values of $r$ one gets small values of the correlation time $\theta$, which indicates that the signal amplitude is strongly influenced by the pump fluctuations and consequently turns out to be incoherent. This in particular is the case when the parametric process takes place with a negligible convection $(\Delta v=0)$ where there is no means for the emergence of a coherent signal, as is the case for the degenerate three-wave interaction also analyzed in [12]. Conversely, for $r \ll 1$, which paradoxally corresponds to the incoherent regime of interaction where the RPA approximation is habitually used, one obtains within the phase-amplitude model the generation of a coherent signal field. This 
feature may be easily interpreted by considering that a strong convection between the signal and the comoving pump and idler waves is responsible for an averaging process in which the signal is no longer sensitive to the pump fluctuations. Moreover, we may notice that $r$ is proportional to the pump correlation time $\tau_{c}$, which means that the coherence of the signal increases as the coherence of the pump decreases. This merely confirms the intuitive idea that the process of convection-induced averaging is more efficient if the pump coherence time $\tau_{c}$ is shorter.

Because the matching of the pump and idler velocities in an actual physical system can never be achieved exactly, the criterion (2) plays an essential role to find the relevant experimental conditions required for the observation of the mixed coherent-incoherent regime of interaction. Inequalities (1) and (2) impose severe constraints on the group velocities in an experiment aimed to observe the generation of a coherent signal from an incoherent pump, namely, inequality (1) requires that the parametric interaction takes place in the presence of strong enough convection $\Delta v$ between the pump and the signal waves (or strong enough temporal walk-off), and inequality (2) requires good matching between pump and idler group velocities.

We have already proposed two experimental configurations in type II singly resonant IOPOs [13] and we show in this paper the feasibility of coherent signal generation from an incoherent cw pump in a type I \{eee $\}$ singly resonant Ti:LiNbO $\mathrm{IOPO}_{3}$. The aspects will be discussed in further details in Sections 2-4. In the SR-IOPO, the resonator is storing and averaging the photons of the resonator signal wave, whereas the nonresonant idler wave will be emitted just after generation at the end of a single pass flight.

We will see that, in order to obtain a net linewidth reduction - or gain of coherence of the signal from the broad-bandwidth pump in the OPO cavity, due to the convectioninduced phase-locking mechanism, inequality (1) involves a simple relation between the walk-off $\Delta v$ and the pump bandwidth $\Delta \nu_{p}$ namely, $\Delta v / v_{p}>\mathrm{FSR} / \Delta \nu_{p}$, where (FSR) is the free spectral range of the signal cavity. If this inequality is satisfied and the pump and idler group velocities are almost matched, only a narrow single mode signal will be build up. Therefore, the broader the pump the stronger the coherence transfer. The influence of the pump bandwidth and group velocity effects in nanosecond OPAs and OPOs have 
been recently explored by Smith [19], leading to similar qualitative results.

\section{Determination of SR-IOPO points of operation}

Our goal is to test the phase-locking mechanism in a standard single pass pump SRIOPO with forward nonlinear coupling. Though critical, inequalities (1) and (2) can be satisfied by exploiting the dispersion properties of a periodically poled Ti:LiNbO 3 waveguide [20, 21]. We consider a type I $\{e e e\}$ nondegenerate three-wave configuration. By selecting a specific quasi-phase-matching (QPM) wavelength triple it is possible to have a negligible group velocity difference between pump (at $\lambda_{p}=1.521 \mu \mathrm{m}$ or at $\lambda_{p}^{*}=$ $1.535 \mu \mathrm{m}$ ) and idler waves (at $\lambda_{i}=2.477 \mu \mathrm{m}$ or at $\lambda_{i}^{*}=2.514 \mu \mathrm{m}$ ), while preserving a considerably different group velocity of the signal [at $\lambda_{s}=\lambda_{p} \lambda_{i} /\left(\lambda_{i}-\lambda_{p}\right)=3.941 \mu \mathrm{m}$ ]. This is illustrated in the group velocity dispersion curve presented in Fig.1, where the selected wavelength triple is marked.

In the following the parameters for the QPM three-wave nonlinear interaction are summarized: [The wavelengths denoted by an asterisk allow amplification of the pump in an Er-doped fiber amplifier; though the pump-idler group velocity matching is worse, the phase-locking mechanism is still efficient. Note, that the signal wavelength was kept constant resulting in a slightly different domain periodicity to get QPM.]

$$
\begin{gathered}
\lambda_{p}=1.52121 \mu \mathrm{m} ; \quad n_{p}=2.14502 ; \quad v_{p} / c=0.455796 ; \quad \alpha_{p}=0.023 \mathrm{~cm}^{-1} \\
{\left[\lambda_{p}^{*}=1.5350 \mu \mathrm{m} ; \quad n_{p}^{*}=2.14458 ; \quad v_{p}^{*} / c=0.455871 ; \quad \alpha_{p}^{*}=0.023 \mathrm{~cm}^{-1}\right]} \\
\lambda_{i}=2.47731 \mu \mathrm{m} ; \quad n_{i}=2.11640 ; \quad v_{i} / c=v_{p} / c-2 \times 10^{-7} ; \quad \alpha_{i}=0.023 \mathrm{~cm}^{-1} \\
{\left[\lambda_{i}^{*}=2.51410 \mu \mathrm{m} ; \quad n_{i}^{*}=2.11524 ; \quad v_{i}^{*} / c=v_{p}^{*} / c-4 \times 10^{-4} ; \quad \alpha_{i}^{*}=0.023 \mathrm{~cm}^{-1}\right]} \\
\lambda_{s}=3.94152 \mu \mathrm{m} ; \quad n_{s}=2.06027 ; \quad v_{s} / c=0.445525 ; \quad \alpha_{s}=0.060 \mathrm{~cm}^{-1}
\end{gathered}
$$

where $\alpha_{j}=2 \gamma_{j} / v_{j}$ is the intensity spatial damping of the respective waves, corresponding to a power loss coefficient of $0.1 \mathrm{~dB} / \mathrm{cm}$ for the pump and idler waves, and of $0.26 \mathrm{~dB} / \mathrm{cm}$ 
for the mid-infrared signal [23]. The required QPM grating periodicities are $\Lambda_{\mathrm{QPM}}=$ $30.2556 \mu \mathrm{m}$ and $\Lambda_{\mathrm{QPM}}^{*}=30.2478 \mu \mathrm{m}$, respectively.

The temporal walk-off is given by the group velocity difference between the pump (or idler) wave and the signal wave:

$$
\Delta v / v_{p}=\left|v_{s}-v_{p}\right| / v_{p}=0.02253 \simeq 1 / 44
$$

\section{Singly resonant integrated optical parametric oscillator}

To simulate the planned experiment we have to consider the integrated optical parametric oscillator (IOPO) in the Fabry-Perot configuration of length $L$. We only take into account the signal amplitude reflectivity at each waveguide face $\rho_{s}=\sqrt{R}$ characterizing the singly resonant integrated OPO (SR-IOPO) $\left(\rho_{p}=\rho_{i}=0\right)$.

The resonant conditions for the guided Fabry-Perot configuration are:

$$
\omega_{p}=\omega_{s}+\omega_{i} ; \quad k_{p}-k_{s}-k_{i}=K
$$

where $K=2 \pi / \Lambda_{\mathrm{QPM}}$, and $\Lambda_{\mathrm{QPM}}$ is the grating pitch for the quasi-phase matching.

We solve a $2 \times$ three-wave equation model for the spatiotemporal evolution of the slowly varying envelopes circulating in the resonator:

$$
\begin{gathered}
\left(\partial_{t} \pm v_{p} \partial_{z}+\gamma_{p}+i \beta_{p} \partial_{t t}\right) A_{p \pm}=-\sigma_{p} A_{s \pm} A_{i \pm} \\
\left(\partial_{t} \pm v_{s} \partial_{z}+\gamma_{s}+i \beta_{s} \partial_{t t}\right) A_{s \pm}=\sigma_{s} A_{p \pm} A_{i \pm}^{*} \\
\left(\partial_{t} \pm v_{i} \partial_{z}+\gamma_{i}+i \beta_{i} \partial_{t t}\right) A_{i \pm}=\sigma_{i} A_{p \pm} A_{s \pm}^{*}
\end{gathered}
$$

where $A_{p, s, i \pm}(z, t)$ stands for the forward $(+)$ or backward $(-)$ propagating wave amplitudes at group velocities $v_{j}(j=p, s, i)$ and temporal damping coefficients $\gamma_{j}$, for the pump, signal, and idler waves, respectively. They are coupled together through the boundary conditions for the Fabry-Perot cavity: 


$$
\begin{gathered}
A_{p+}(0, t)=E_{p}(0) \exp [i \Phi(t)]+g_{n, p} \\
A_{p-}(L, t)=g_{n, p} \\
A_{s+}(0, t)=\rho_{s} A_{s-}(0, t)+g_{n, s} \\
A_{s-}(L, t)=\rho_{s} A_{s+}(L, t)+g_{n, s} \\
A_{i+}(0, t)=g_{n, i} \\
A_{i-}(L, t)=g_{n, i}
\end{gathered}
$$

where $E_{p}(0)$ is the cw pump field amplitude determining the nonlinear characteristic time $\tau_{0}=2 /\left[E_{p}(0) \sigma_{p}\right]$ of the parametric three-wave interaction through the nonlinear coupling coefficient $\sigma_{p}, \phi$ a randomly Gaussian distributed phase, and $g_{n, j}$ stands for thermal noises $\left(\left\langle\left|g_{n, j}\right|^{2}\right\rangle \simeq 10^{-10} E_{p}(0)^{2}\right)$. The pump autocorrelation function reads as $\left\langle A_{p}\left(0, t^{\prime}+t\right) A_{p}^{*}(0, t)\right\rangle=E_{p}(0)^{2} \exp \left(-|t| / \tau_{c}\right)$, where $\tau_{c} \simeq 1 / \pi \Delta \nu$ is the correlation time and $\Delta \nu$ is the (broad)-bandwidth of the pump spectrum. Equations (5) include the effect of chromatic dispersion, whose influence has to be taken into account for rapid random phase variations of the input pump. The effects of group velocity dispersion (GVD) are represented by the second derivatives with respect to time, so that the dispersion parameters are given by $\beta_{j}=v_{j} k_{j}^{\prime \prime} / 2$, where $k_{j}^{\prime \prime}=\left(\partial^{2} k / \partial \omega^{2}\right)_{j}, k$ being the wave vector modulus, $k=n(\omega) \omega / c$.

The nonlinear coupling coefficients of Eqs.(5) are

$$
\sigma_{j}=\frac{2 \pi d_{e f f} v_{j} f_{j, k, l}}{\lambda_{j} n_{j}}
$$

where $n_{j}$ is the refractive index at frequency $\omega_{j}, d_{e f f}=2 d / \pi$ is the effective nonlinear susceptibility, and $f_{j, k, l}$ are the dimensionless mode overlap factors which are evaluated in Appendix A. For a nonlinear effective susceptibility $d_{\text {eff }}=2 d / \pi=12.4 \mathrm{pm} / \mathrm{V}$, and overlap factors (A10), the effective nonlinear coupling coefficients (7) of Eqs.(5) are:

$$
\sigma_{p}=2866 \mathrm{~m} /(\mathrm{s} \mathrm{V}) ; \quad \sigma_{i}=940 \mathrm{~m} /(\mathrm{s} \mathrm{V}) ; \quad \sigma_{s}=262 \mathrm{~m} /(\mathrm{s} \mathrm{V})
$$




\section{Dynamics of the incoherently driven SR-IOPO}

The power threshold of the SR-IOPO is determined by the effective nonlinearity, the

cavity length $L$, the propagation losses $\gamma_{j}$ (or $\alpha_{j}$, respectively), and the mirror reflectivity $R=\rho_{s}^{2}$; it is independent on the pump bandwidth if the group velocity of the pump matches the group velocity of the idler. Therefore, as we show hereafter, it coincides with the SR-IOPO threshold for a coherent pump [6] [8].

We assume a pump with a Gaussian random phase fluctuating with a correlation time $\tau_{c}$. These phase fluctuations induce amplitude fluctuations during propagation in the waveguide due to dispersion. One of the main results of [12] has been to show that the parametric gain of the nondegenerate three-wave interaction (and therefore the OPO threshold) is exactly the same for a coherent pump as for a random phase fluctuating pump, provided that the group velocities of pump and idler are matched. Indeed, in this case the idler completely absorbs the rapid phase fluctuations of the pump and the signal experiences a parametric gain insensitive to the pump fluctuations. The reason is that the pump-idler phase fluctuations are locked together. In [12] it is also proved that it is not necessary to have an exact group velocity match; a group velocity difference smaller than the pump correlation length times the idler damping time is sufficient. This condition is equivalent to inequality (2). However, in order to obtain a net gain of coherence for the signal, another condition is required, namely, a large enough convection of the signal with respect to the pump-idler motion (i.e. large enough temporal walk-off), which is expressed by inequality (1). This group velocity condition is developed in [12] by calculating the autocorrelation function.

The parametric gain is related to the nonlinear characteristic time $\tau_{0}$ and the corresponding nonlinear characteristic length $L_{n l}$, given by

$$
\tau_{0}=\frac{2}{E_{p}(0) \sigma_{p}}=0.181 \mathrm{~ns} ; \quad L_{n l}=v_{p} \tau_{0}=2.47 \mathrm{~cm}
$$

The physical cavity length we have chosen, namely, $L=8.00 \mathrm{~cm}$, determines the dimensionless cavity length $\Lambda_{n l}=L / L_{n l}=3.24$, which measures the amplitude gain length. It is introduced in the dimensionless system of equations used to perform the simulations 
( $c f$ Appendix B); $\Lambda_{n l}$ is a function of the input pump power through definitions (9).

\section{A. Threshold and power characteristics:}

For the above effective cross section and cavity length parameters and assuming exact quasi-phase matching in the waveguide cavity, the threshold of the singly resonant $\left(R=\rho_{s}^{2}=98.01 \%\right) \mathrm{Ti}: \mathrm{LiNbO}_{3}$ IOPO is reached at a pump power $P_{\text {thres }}=1250 \mathrm{~mW}$ (which corresponds to the dimensionless length $\Lambda_{n l}=3.17$ ). We have analyzed a large pump power range for this mirror reflectivity. The resulting power characteristic is plotted in Fig.2 as signal and idler output power versus input pump power. A spectral width of the pump of $\Delta \nu_{p}=86.10 \mathrm{GHz}$ has been assumed; the associated pump correlation time is $\tau_{c}=\left(\pi \Delta \nu_{p}\right)^{-1}=3.69$ ps. Figure 2 also shows that the power characteristics for a coherent pump (denoted by the + symbols) coincide with those of a broad-bandwidth pump independent of the pump linewidth. The threshold, numerically determined from Eqs.(5), coincides with the standard analytical formula for SR-IOPOs [6]. Therefore it is straight off to obtain similar power characteristics for other mirror reflectivities by using this coherent SR-IOPO threshold formula.

For the chosen mirror reflectivity $(R=98.01 \%)$ up to a pump power of $1570 \mathrm{~mW}$, inequality (1) remains satisfied since the nonlinear characteristic time $\tau_{0}\left(P_{p}=1570 \mathrm{~mW}\right)=$ 0.164 ns yields

$$
\frac{\tau_{c}^{e f f}}{\tau_{0}}=\frac{v_{p}}{\Delta v} \frac{\tau_{c}}{\tau_{0}}=0.998<1
$$

\section{B. Dynamics:}

We have performed dynamical simulations for a pump power of $1300 \mathrm{~mW}$. At this level the internal conversion efficiency for the signal is $1.4 \%$, defined as the ratio of the average signal power inside the cavity with respect to the average input pump power [yielding $2.8 \times 10^{-4}$ external conversion efficiency]. The conversion efficiency for the idler is $3.3 \%$ (see Fig.2). The dynamics of the SR-IOPO is shown in Figs.3-6.

The incoherent cw pump of correlation time $\tau_{c}=(\pi \Delta \nu)^{-1}=0.0204 \tau_{0}=3.69 \mathrm{ps}$ is

well fitted by a Lorentzian spectral profile of FWHM bandwidth $\Delta \nu_{p}=\left(\pi \tau_{c}\right)^{-1}=86.10$ $\mathrm{GHz}\left(\Delta \lambda_{p}=0.663 \mathrm{~nm}\right)$, much larger than the $(\mathrm{FSR})=c /\left(2 n_{s} L\right)=910 \mathrm{MHz}$ of the 
Fabry-Perot cavity. The dynamics of pump, idler, and signal evolution in the waveguide (cavity) is strongly influenced by the crystal dispersion $\left(k^{\prime \prime}=0.1 \mathrm{ps}^{2} / \mathrm{m}\right)$, which couples the evolution of the phases to that of the intensities and transforms the random phase fluctuations into intensity fluctuations. However, the dispersion does not destroy the convection-induced phase-locking mechanism, which requires a characteristic dispersion length $L_{d}=\tau_{c}^{2} /\left(2 \beta_{j}\right)$, much larger than the nonlinear characteristic length $L_{n l}=v_{p} \tau_{0}$ [12]. Inequalities (1) and (2) are satisfied, since we have:

$$
\Im_{1} \equiv \frac{\tau_{c}^{e f f}}{\tau_{0}}=\frac{v_{p}}{\Delta v} \frac{\tau_{c}}{\tau_{0}}=0.905<1, \quad \Im_{2} \equiv \frac{\left|v_{i}-v_{p}\right|}{v_{p}} \frac{1}{\tau_{c} \gamma_{i}}=2 \times 10^{-4}<1 .
$$

[Note, that for the wavelengths with a star in Eq.(3) one gets $\Im_{2}=0.78<1$ ].

Starting from a random noise for signal and idler, the incoherent pump amplifies both. Because of the small group velocity difference between the pump and the idler waves [cf. second expression (10)] and appreciable convection (4) of the pump with respect to the signal, the phase-locking mechanism takes place efficiently: the incoherence of the pump is transferred to the idler wave allowing the signal wave to grow efficiently with a high degree of coherence.

Figure 3 shows the spatial evolution of the amplitudes in the SR-IOPO of $8 \mathrm{~cm}$ length at $t=16384 t_{r}=18 \mu \mathrm{s}$, where the round-trip time in the Fabry-Perot cavity is $t_{r}=2 n_{s} L / c=1 / \mathrm{FSR}=1.098 \mathrm{~ns}\left(\simeq 6.07 \tau_{0}\right)$. The incoherent, random phase modulated pump at the input $(\mathrm{z}=0)$ generates strong amplitude fluctuations during propagation to the output due to dispersion in the waveguide (upper graph). As the pump level is above threshold, signal and idler waves are generated. It can be observed that the pump fluctuations are transferred to the growing (nonresonant) idler only, whereas the resonant signal has nearly a constant amplitude. The fluctuations of the pump are transferred to the idler wave moving at the same group velocity, whereas the resonant signal experiences a temporal walk-off and exhibits small fluctuations only. Both the relative average modulation amplitudes of pump and idler fluctuations are exactly the same as can be seen in Fig.4(a)-4(b) and correspond to the same broad bandwidth as shown in Figs. 5(a)-5(b). 
The relative average fluctuating amplitudes are defined by

$$
\left\langle\Delta\left|A_{j}(L, t)\right|\right\rangle \equiv \frac{\left[\left\langle\left|A_{j}(L, t)\right|^{2}\right\rangle-\left\langle\left|A_{j}(L, t)\right|\right\rangle^{2}\right]^{1 / 2}}{\left\langle\left|A_{j}(L, t)\right|\right\rangle}, \quad(j=p, s, i),
$$

yielding asymptotically

$$
\left\langle\Delta\left|A_{p}(L, t)\right|\right\rangle=0.2349, \quad\left\langle\Delta\left|A_{i}(L, t)\right|\right\rangle=0.2356, \quad\left\langle\Delta\left|A_{s}(L, t)\right|\right\rangle=0.01708,
$$

These numbers correspond to a reduction of the average signal intensity fluctuations $\left\langle\Delta I_{s}(L, t)\right\rangle \equiv\left\langle\Delta\left|A_{s}(L, t)\right|^{2}\right\rangle$ with respect to the average pump intensity fluctuations $\left\langle\Delta I_{p}(L, t)\right\rangle \equiv\left\langle\Delta\left|A_{p}(L, t)\right|^{2}\right\rangle$ by almost 3 orders of magnitude.

The significant reduction of the signal fluctuations, compared to the pump and idler fluctuations, is accompanied by a gain of coherence as shown by the spectral density of Fig.5(a)-5(d), evaluated at time $t=16384 t_{r}=18 \mu \mathrm{s}$, when the efficient coherence transfer has attained the asymptotic stage. We observe that almost all the incoherence of the pump [Fig.5(a)] has been transmitted to the idler wave [Fig.5(b)]; the signal wave emerges with a single line bandwidth $\Delta \nu_{s}$ less than $2 \times 10^{-5} \Delta \nu_{p}$ [Fig.5(c) and Fig.5(d)]. This signal line bandwidth may be as narrow as that given by the Shawlow-Townes limit [24], whatever the broad bandwidth pump resulting from the random phase fluctuating phase. However we are not able to get it within the limited resolution of our spectral analysis $(\mathrm{FSR} / 512 \simeq 1.7 \mathrm{MHz})$.

The attractor tendency to coherence during the temporal evolution is shown in logarithmic scale in Fig.6(a)-6(d). At the transient time $t=4096 t_{r}$ the signal spectrum still presents low satellites [Fig.6(a)], indicating a small transient modulation at the FSR frequency. At time $t=16384 t_{r}$ this modulation has relaxed to a very low level [Fig.5(c)], quantitatively evident in logarithmic scale [Fig.5(d) or 6(d)].

\section{Dynamics for a pump shifted to $\lambda_{p}=1.535 \mu \mathrm{m}$}

We have also analyzed the dynamics of the SR-IOPO operated by a pump of slightly shifted wavelength to $\lambda_{p}=1.535 \mu \mathrm{m}$. This wavelength would allow amplification of the pump in an Er-doped fiber amplifier. The QPM idler wavelength is $\lambda_{i}=2.51410 \mu \mathrm{m}$ 
with a resonant signal again at $\lambda_{s}=3.94152 \mu \mathrm{m}$ using a grating pitch slightly modified to $\Lambda_{\mathrm{QPM}}=30.2478 \mu \mathrm{m}$. Now, the pump-idler group velocity mismatch is increased to $\delta v / v=4.65 \times 10^{-4}$, but inequality (2) still holds, since $\tau_{c} \gamma_{i}=5.9 \times 10^{-4}$ and Eq.(10) yields $\Im_{2}=0.78<1$. Therefore, we can also expect an efficient convection-induced phase-locking effect. However, it takes longer before the asymptotic regime is achieved. Indeed, until $32768 t_{r}$ the signal still remains multimode and it tends to a single line above $40,000 t_{r}$. The asymptotic regime bifurcates to a coherent attractor, and we obtain at $49152 t_{r}$ (for the same pump power and pump bandwidth), the same spectral coherence transfer as in Subsection 4.B, namely, $\Delta \nu_{s}<2 \times 10^{-5} \Delta \nu_{p}$. This gain of coherence is accompanied by a smaller reduction of the signal fluctuations compared to the pump and idler fluctuations. The relative average fluctuating amplitudes at the asymptotic state are

$$
\left\langle\Delta\left|A_{p}(L, t)\right|\right\rangle=0.2365, \quad\left\langle\Delta\left|A_{i}(L, t)\right|\right\rangle=0.2305, \quad\left\langle\Delta\left|A_{s}(L, t)\right|\right\rangle=0.02519,
$$

which correspond to a reduction of the average signal intensity fluctuations $\left\langle\Delta I_{s}(L, t)\right\rangle$ with respect to the average pump intensity fluctuations $\left\langle\Delta I_{p}(L, t)\right\rangle$ by a factor of only $10^{-2}$.

\section{Pump bandwidth dependence and robustness of the convection- induced phase-locking coherence transfer}

Let us consider the pump bandwidth dependence of the coherence transfer in order to illustrate the paradoxal effect that the larger the pump bandwidth is, while preserving the nonoverlap of the three wave spectra (pump, idler, and signal), the better works the convection-induced phase-locking mechanism and the greater is the linewidth reduction. Once inequality (2) is satisfied, i.e. good group velocity matching between pump and idler waves, inequality (1) yields a simple relation between the relative group velocity convection $\Delta v / v_{p}$ of the signal with respect to the pump, and the pump bandwidth $\Delta \nu_{p} \simeq\left(\pi \tau_{c}\right)^{-1}$, namely,

$$
\frac{\Delta v}{v_{p}}>\frac{1}{\pi \Delta \nu_{p} \tau_{0}}
$$

If this inequality is well-satisfied only a narrow single mode signal is built up from the 
broad-bandwidth pump. Therefore, the broader the pump the larger the gain of coherence. However, when inequality (14) is not satisfied, the signal is able to follow the pump fluctuations and even if the parametric gain is not changed, the signal loses the high coherence and its spectrum can become multiline.

To test the robustness of the convection-induced phase-locking coherence transfer ensuring the experimental feasibility, we have performed simulations well above threshold in all the pump power range of Fig.2. Higher pump power leads to shorter $\tau_{0}$ and increases the right hand side of inequality (14). Let us choose a higher pump power point of operation as that considered in Subsection 4.B, namely, $P_{p}=1520 \mathrm{~mW}\left(\tau_{0}=0.167 \mathrm{~ns}\right)$. The characterisic values for a set of eight different pump bandwidths are reported in Table 1. Let us comment here three qualitatively different cases (a.), (c.) and (h.):

(a.) a broad pump of linewidth $\Delta \nu_{p}=140.36 \mathrm{GHz} ;\left(\Delta \lambda_{p}=1.08 \mathrm{~nm}\right)$;

(c.) a pump of linewidth $\Delta \nu_{p}=67.69 \mathrm{GHz} ;\left(\Delta \lambda_{p}=0.522 \mathrm{~nm}\right)$; and

(h.) a narrow pump, or coherent light beam, of linewidth $\Delta \nu_{p}=2.11 \mathrm{GHz} ;\left(\Delta \lambda_{p}=\right.$ $0.0163 \mathrm{~nm})$.

As we could guess, case (a.), which well-verifies inequality (14), namely, $\Delta v / v=$ $0.02253>0.0135=\left(\pi \Delta \nu_{p} \tau_{0}\right)^{-1}$, exhibits a high coherence transfer, namely, $\Delta \nu_{s} / \Delta \nu_{p}<$ $1.2 \times 10^{-5}$ and a net reduction of the mean intensity fluctuations of the signal with respect to the pump $\left\langle\Delta I_{s}\right\rangle /\left\langle\Delta I_{p}\right\rangle=2.8 \times 10^{-3}$, as we can read on Table 1 . We obtain similar spectra as those presented in Section 4. Case (c.) violates inequalities (1) or (14) and gives rise to a multimode signal [Figs.7(c) and $7(\mathrm{~d})]$. We can conclude that for this intermediate case the convection-induced phase-locking mechanism does not satisfactorily work. Below this case, even if the mechanism is not efficient since only inequality (2) is verified, the high finesse singly resonant OPO commands a single mode signal. Finally, for the coherent pump case (h.), whose bandwidth still measures about 2 FSR, the resulting signal is also single mode, but this is because only one mode experiences a sufficient gain, independently of the convection-induced phase-locking mechanism. However, to test the sensibility of the coherent case to small pump perturbations, we have also considered the situation where the very narrow pump is perturbed by a slowly periodical modulation 
over a range of $4 \mathrm{FSR}$. The slow modulation is automatically transferred to the signal, the idler wave being unable to lock this modulation, since the effective pump correlation time in this case $\tau_{c}^{e f f} \simeq 6.7 \mathrm{~ns}$ is longer than the nonlinear characteristic time $\tau_{0}=0.167$ ns, and the signal is able to follow the slow perturbation.

The very interesting result is the insensitivity of the signal against such kind of perturbations when acting on a broad-bandwidth pump [as case (a.)]. Indeed, we have performed simulations for different kinds of slowly varying phase and/or amplitude fluctuations over a range of several FSR, and we obtain the same gain of coherence. This is not so amazing since the broad incoherent spectrum contains such a range of frequencies.

\section{Conclusion}

Incoherently cw-pumped singly resonant integrated OPO, in the type I $\{e e e\}$ configuration in Ti:LiNbO 3 waveguides, has been considered for generation of coherent signal output through the convection-induced phase-locking mechanism. The SR-IOPO at $\lambda_{s}=3.94 \mu \mathrm{m}$, exhibiting a group velocity difference $\Delta v / v_{p}=\left|v_{s}-v_{p}\right| / v_{p} \simeq 1 / 44$ with respect the pump and idler waves, has been compared to alternative SR-IOPOs at different resonant frequencies and different temporal walk-off, and has been retained as a good compromise for the experimental realization. All the simulations have been done for different signal dampings in a large range of input pump power. Let us note from inequality (14) that a simple rule for the reduction of a broad bandwidth pump measuring $N$ FSR to a single mode signal, is to have a relative convection $\Delta v / v_{p}$ greater than $N^{-1}$. Then the SR-IOPOs support the fact of a sufficient temporal walk-off for an efficient phase-locking mechanism. The reduction of almost 3 orders of magnitude for the average signal intensity fluctuations with respect to the pump fluctuations and a signal bandwidth smaller than $10^{-5}$ the pump bandwidth have been obtained in the asymptotic stage after less than $20 \mu$ s. This might allow developing MIR-IOPOs of narrow linewidth output for spectroscopy pumped by low coherence cheap semiconductor lasers. 


\section{Appendix A}

The amplitudes $A_{j}$ entering Eqs.(5) are related to the electric fields by

$$
E_{j}(\mathbf{r}, t)=\frac{F_{j}(x, y)}{F_{j, \max }} A_{j}(z, t) \exp \left(i k_{j} z\right)
$$

where $F_{j}(x, y)$ are the respective transverse distributions

$$
F_{j}(x, y)=\sqrt{\frac{2}{\sqrt{\pi} w_{j}}} \exp \left[-2\left(\frac{x}{w_{j}}\right)^{2}\right] \cdot \frac{2}{\sqrt{\sqrt{\pi} d_{j}}} \frac{y}{d_{j}} \exp \left[-\frac{1}{2}\left(\frac{y}{d_{j}}\right)^{2}\right]
$$

approximated by the Gauss-Hermite-Gauss-model; $w_{j}$ and $d_{j}$ are the mode widths and depths, respectively, related to the full width at half maximum (FWHM) of the mode intensity distributions in both dimensions via $\mathrm{FWHM}_{x}=\sqrt{\ln 2} w \simeq 0.832 w$, and $\mathrm{FWHM}_{y} \simeq$ $1.155 d$, respectively; where the relation between $F_{j}(x, y)$ and $F_{j, \max }$ is constructed as: $\iint F_{j}(x, y)^{2} d x d y=F_{j, \max }^{2} S_{j, e f f}=1$.

In this way, the effective mode cross sections are given by

$$
S_{j, e f f}=\frac{1}{F_{j, \max }^{2}}=\frac{e}{8} \pi w_{j} d_{j}
$$

yielding with the data given in Eqs.(3) the respective transverse sections,

$$
S_{p}=30.99 \mu \mathrm{m}^{2}, \quad S_{i}=58.78 \mu \mathrm{m}^{2}, \quad S_{s}=133.15 \mu \mathrm{m}^{2},
$$

associated with the wave powers,

$$
P_{j}=I_{j} S_{j, e f f}=\frac{1}{2} n_{j} \varepsilon_{0} c\left|A_{j}\right|^{2} S_{j, e f f},
$$

where $I_{j}=(1 / 2) n_{j} \varepsilon_{0} c\left|A_{j}\right|^{2}$ are the flux intensities.

The nonlinear coupling coefficients of Eqs.(5) are

$$
\sigma_{j}=\frac{2 \pi d_{e f f} v_{j} f_{j, k, l}}{\lambda_{j} n_{j}}
$$

where $n_{j}$ is the refractive index at frequency $\omega_{j}, d_{\text {eff }}=2 d / \pi$ is the effective nonlinear susceptibility, and $f_{j, k, l}$ is the dimensionless mode overlap factor:

$$
f_{j, k, l}=\frac{F_{j, \max }}{F_{k, \max } F_{l, \max }} \iint \mathrm{dx} \operatorname{dy} F_{j}(x, y) F_{k}(x, y) F_{l}(x, y) .
$$


For the given resonant set of frequencies Eqs.(3), the overlap integral yields

$$
\iint \mathrm{dx} \operatorname{dy} F_{p}(x, y) F_{i}(x, y) F_{s}(x, y)=55265.1 \mathrm{~m}^{-1}
$$

and from Eqs.(A3) and (A4) the respective factors entering Eq.(A7) yield

$$
\frac{F_{p, \max }}{F_{i, \max } F_{s, \max }}=15.89 \mu \mathrm{m} ; \quad \frac{F_{i, \max }}{F_{p, \max } F_{s, \max }}=8.378 \mu \mathrm{m} ; \quad \frac{F_{s, \max }}{F_{p, \max } F_{i, \max }}=3.689 \mu \mathrm{m}
$$

Therefore, from Eqs.(A7), (A8) and (A9), we obtain the dimensionless mode overlap factors $f_{j} \equiv f_{j, k, l}$ which reduce the nonlinear coupling coefficients in the guiding medium:

$$
f_{p}=0.8781, \quad f_{i}=0.4630, \quad f_{s}=0.2043
$$

For a nonlinear effective susceptibility $d_{\text {eff }}=2 d / \pi=12.4 \mathrm{pm} / \mathrm{V}$, and overlap factors (A10), we evaluate the nonlinear coupling coefficients (A6) of Eqs.(5):

$$
\sigma_{p}=2866 \mathrm{~m} /(\mathrm{s} \mathrm{V}) ; \quad \sigma_{i}=940 \mathrm{~m} /(\mathrm{s} \mathrm{V}) ; \quad \sigma_{s}=262 \mathrm{~m} /(\mathrm{s} \mathrm{V})
$$




\section{Appendix B}

Let us rewrite Eqs.(5) for the forward propagating wave amplitudes in order to present the dimensionless system of equations used for the numerical simulations:

$$
\begin{aligned}
\left(\partial_{t}+v_{p} \partial_{z}+\gamma_{p}+i \beta_{p} \partial_{t t} A_{p}\right. & =-\sigma_{p} A_{s} A_{i} \\
\left(\partial_{t}+v_{s} \partial_{z}+\gamma_{s}+i \beta_{s} \partial_{t t}\right) A_{s} & =\sigma_{s} A_{p} A_{i}^{*} \\
\left(\partial_{t}+v_{i} \partial_{z}+\gamma_{i}+i \beta_{i} \partial_{t t}\right) A_{i} & =\sigma_{i} A_{p} A_{s}^{*}
\end{aligned}
$$

The dimensionless variables are defined by introducing the nonlinear characteristic time and length,

$$
\tau_{0}=\frac{2}{E_{p}(0) \sigma_{p}}, \quad L_{n l}=v_{p} \tau_{0}
$$

namely,

$$
\tau=\frac{t}{\tau_{0}}, \quad \zeta=\frac{z}{L_{n l}}, \quad \mu_{j}=\gamma_{j} \tau_{0}
$$

and normalizing the amplitudes to the coherent input pump amplitude $E_{p}(0)$ through

$$
a_{j}=\frac{A_{j}}{E_{p}(0)} .
$$

They take the form

$$
\begin{gathered}
\left(\partial_{\tau}+\partial_{\zeta}+\mu_{p}+i \tilde{\beta}_{p} \partial_{\tau \tau}\right) a_{p}=-2 a_{s} a_{i} \\
\left(\partial_{\tau}+\partial_{\zeta}+\mu_{s}+i \tilde{\beta}_{s} \partial_{\tau \tau}\right) a_{s}=\kappa_{s} a_{p} a_{i}^{*} \\
\left(\partial_{\tau}+\partial_{\zeta}+\mu_{i}+i \tilde{\beta}_{i} \partial_{\tau \tau}\right) a_{i}=\kappa_{i} a_{p} a_{s}^{*} \\
\kappa_{s}=2 \sigma_{s} / \sigma_{p}=0.1827 ; \quad \kappa_{i}=2 \sigma_{i} / \sigma_{p}=0.6561 ;
\end{gathered}
$$

where $\tilde{\beta}_{j}$ is the dimensionless dispersion coefficient. The parametric gain is related to the nonlinear characteristic time $\tau_{0}$ and the corresponding nonlinear characteristic length $L_{n l}$. 
To investigate the IOPO we fix the pump power and cavity length and increase the cavity finesse until the gain exceeds the cavity losses. For a pump power $P_{p}=1300 \mathrm{~mW}$ launched into an effective pump cross section $S_{p}=31 \mu \mathrm{m}^{2}$, yielding a pump intensity flux $I_{p}=P_{p} / S_{p}=4.193 \mathrm{MW} / \mathrm{cm}^{2}$, which determines the input pump field amplitude $E_{p}(0)[\mathrm{MV} / \mathrm{m}]=\left[I_{p}(0)\left[\mathrm{MW} / \mathrm{cm}^{2}\right] / 0.2838\right]^{1 / 2}=3.843 \mathrm{MV} / \mathrm{m}$, and for a cavity length $L=8.00 \mathrm{~cm}$, we obtain a comfortable point of operation above threshold for a signal amplitude reflectivity $\rho_{s}=0.99$ (or mirror intensity reflectivity $R=98.01 \%$ ). The simulations shown in Figs.2-7 correspond to a signal damping $\alpha_{s}=0.06 \mathrm{~cm}^{-1}$.

For the pump power $P_{p}=1300 \mathrm{~mW}$ we have

$$
\tau_{0}=\frac{2}{E_{p}(0) \sigma_{p}}=0.181 \mathrm{~ns}, \quad L_{n l}=v_{p} \tau_{0}=2.47 \mathrm{~cm}
$$

The chosen physical cavity length determines the dimensionless cavity length $\Lambda_{n l}=$ $L / L_{n l}=3.24$ we must take for the numerical simulations of the dimensionless system of Eqs. (B7); it is a function of the input pump power through definitions (B8): $\Lambda_{n l}\left(P_{p}\right)=3.24\left(P_{p}[\mathrm{~mW}] / 1300 \mathrm{~mW}\right)^{1 / 2}$.

As we can see on Fig.2, the power threshold (for $R=0.9801$ ) is obtained for

$$
P_{p}=1250 \mathrm{~mW}, \quad \tau_{0}=0.185 \mathrm{~ns}, \quad L_{n l}=2.52 \mathrm{~cm} ; \quad L=\Lambda_{n l} L_{n l}=8.00 \mathrm{~cm}
$$

which corresponds to the dimensionless length and loss parameters,

$$
\Lambda_{n l}=L / L_{n l}=3.17, \quad \mu_{p}=\mu_{i}=0.028, \quad \mu_{s}=0.074
$$

used in Eqs.(B7). 


\section{Appendix C}

The role of convection in the coherence of the generated waves $A_{s, i}$ can be obtained by considering a dispersionless interaction $\left[\beta_{j}=0\right.$ in Eqs.(5) or Eqs.(B1)-(B3)] in the linear limit of its evolution or parametric approximation (without pump depletion). We assume for simplicity $\sigma_{s}=\sigma_{i}=\sigma_{p} / 2$. In this limit, if pump losses are neglected, the incoherent pump wave is modeled, in the reference frame traveling at its group velocity, $z=x-v_{p} t$, by a stationary single-variable stochastic function $A_{p}(z)$ that we will characterize through its coherence length $\lambda_{c}=\tau_{c} v_{p}$. Integrating Eq.(B3) along the characteristic of the idler wave and substituting the solution in Eq.(B1), we have

$$
D A_{s}=\sigma^{2} \int_{0}^{t} e^{-\gamma_{i}\left(t-t^{\prime}\right)} A_{p}(z) A_{p}^{*}\left(z^{\prime}\right) A_{s}\left(x^{\prime}, t^{\prime}\right) \mathrm{d} t^{\prime}
$$

where $D=\partial / \partial t+v_{s} \partial / \partial x+\gamma_{s}, z^{\prime}=z-\left(v_{i}-v_{p}\right)\left(t-t^{\prime}\right)$, and $x^{\prime}=x-v_{i}\left(t-t^{\prime}\right)$. The presence of the factor $A_{p}(z) A_{p}^{*}\left(z^{\prime}\right)$ in the integrand of Eq. $(\mathrm{C} 1)$ reveals the existence of a particular regime of interaction. Indeed, in the situation where the idler and pump velocities are equal, $v_{i}=v_{p}$, one has $z^{\prime}=z$, and this factor becomes $\left|A_{p}(z)\right|^{2}$, which shows that the signal evolution is independent of the pump phase fluctuations $\Phi_{p}(z)$. This result suggests the possibility to generate, from an incoherent pump, a signal with slow phase variations, i.e. a signal with a high degree of coherence. This phenomenon can be understood through the analysis of the idler wave, whose behavior is given by the solution of Eq.(B3) :

$$
A_{i}(x, t)=\sigma \int_{0}^{t} e^{-\gamma_{i}\left(t-t^{\prime}\right)} A_{p}\left(z^{\prime}\right) A_{s}^{*}\left(x^{\prime}, t^{\prime}\right) \mathrm{d} t^{\prime}
$$

When $v_{i}=v_{p}$ one has $z^{\prime}=z$ so that the function $A_{p}\left(z^{\prime}\right)$ becomes independent of time $t^{\prime}$ and we can remove it from the integral, thus showing that the idler field is directly proportional to the pump field. Let us notice that this pump-idler phase-locking mechanism does not require an exact velocity matching $v_{i}=v_{p}$. Indeed, it is sufficient that the velocities obey the inequality

$$
\left|v_{i}-v_{p}\right| \ll \lambda_{c} \gamma_{i}=\tau_{c} v_{p} \gamma_{i}
$$


to be able to remove the amplitude $A_{p}\left(z^{\prime}\right)$ from the integral so that the idler follows the pump phase fluctuations. Inequality (C3) is nothing else as inequality (2).

To justify inequality (1) we must evaluate the autocorrelation function of the generated signal wave. We will not repeat here the calculations which are developed in [12]. 


\section{References}

[1] See the feature on parametric devices, J. Opt. Soc. Am. B 12, 2084-2320 (1995).

[2] S.T. Yang, R.C. Eckardt, and R.L. Byer, Continuos-wave singly resonant optical parametric oscillator pumped by a single-frequency resonantly doubled Nd:YAG laser, Opt. Lett. 18 971-973 (1993).

[3] S.T. Yang, R.C. Eckardt, and R.L. Byer, Power and spectral characteristics of continuous-wave parametric oscillators: the doubly to singly resonant transition, J. Opt. Soc. Am. B 10, 1684-1695 (1993).

[4] M.E. Klein, D.H.Lee, J.P. Meyn, K.J. Boller, and R. Wallenstein, Singly resonant continuous-wave optical parametric oscillator pumped by a diode laser, Opt. Lett. 24, 1142-1144 (1999).

[5] W. Sohler and H. Suche, in Digest of the Third International Conference on Integrated Optics and Optical Fiber Communication (Optical Society of America, 1981), p.89

[6] G.P. Bava, I. Montrosset, W. Sohler, and H. Suche, Numerical Modeling of Ti:LiNbO 3 Integrated Optical Parametric Oscillators, IEEE J. Quantum Electron. QE-23, 42-51 (1987).

[7] H. Suche and W. Sohler, Integrated optical parametric oscillators (invited), Optoelectronics-Devices and Technologies, Japan, 4(1) p.1-20 (1989).

[8] M.A. Arbore and M.M. Fejer, Singly resonant optical parametric oscillation in periodically poled lithium niobate waveguides, Opt. Lett. 22, 151-153 (1997).

[9] D. Hofmann, H. Herrmann, G. Schreiber, W. Grundkötter, R. Ricken, and W. Sohler, Continuous-wave mid-infrared optical parametric oscillators with periodically poled Ti: $\mathrm{LiNbO}_{3}$ waveguide, in 9th European Conference on Integrated Optics and Technical Exhibition ECIO'99 (EOS - European Optical Society 1999), p. 21. 
[10] D. Hofmann, H. Herrmann, G. Schreiber, C. Haase, W. Grundkötter, R. Ricken, and W. Sohler, in Nonlinear Guided Waves and Their Applications (Optical Society of America, 1999), FC2-1-3, p.465.

[11] A. Picozzi and M. Hælterman, Parametric Three-Wave Soliton Generated from Incoherent Light Phys. Rev Lett. 86, 2010-2013 (2001).

[12] A. Picozzi, C. Montes, and M. Hælterman, Coherence properties of the parametric three-wave interaction driven from an incoherent pump, Phys. Rev E 66, 056605-1-14 (2002).

[13] C. Montes, A. Picozzi, and K. Gallo, Ultra-coherent output from an incoherent cw-pumped singly resonant optical parametric oscillator, Opt. Commun. 237, 437-449 (2004).

[14] V.N. Tsytovich, Nonlinear Effects in Plasma (Plenum, New York, 1970).

[15] V.E. Zakharov, V.S. L'vov and G. Falkovich, Kolmogorov Spectra of Turbulence I, Springer Series in Nonlinear Dynamics, (Springer, 1992).

[16] A. Martins and J.T. Mendonça, Projection-operator method of the nonlinear three-wave interaction. Phys. Rev. A 31, 3898-3906 (1985).

[17] A. Martins and J.T. Mendonç The non-linear three-wave interaction with a finite spectral width, Phys. Fluids 31, 3286-3294 (1988).

[18] R. L. Berger, Suppression of Parametric Instability by Weakly Incoherent Laser Beams Phys. Rev. Lett. 65, 1207-1210 (1990).

[19] A.V. Smith Bandwidth and group velocity effects in nanosecond optical parametric amplifiers and oscillators, J. Opt. Soc. Am. B 22, 1953-1965 (2005).

[20] D. Hofmann, G. Schreiber, W. Grundkötter, R. Ricken, and W. Sohler, Midinfrared continuous-wave singly resonant optical parametric oscillator with periodically poled Ti: $\mathrm{LiNbO}_{3}$ waveguide, Conf. Digest 2000, Conf. on Lasers and Electro-Optics Europe (CLEO'00), Nice, France, september 2000, paper CDM4. 
[21] G. Schreiber, D. Hofmann, W. Grundkötter, Y.L. Lee, H. Suche, V. Quiring, R. Ricken, and W. Sohler, Nonlinear integrated optical frequency converters with periodically poled Ti: $\mathrm{LiNbO}_{3}$ waveguides, Proc. SPIE, vol 4277, 144-160 (2001) Photonics West 2001, paper 4277-18 (invited).

[22] D.E. Zelmon, D.L. Small, and D. Jundt, Infrared corrected Sellmeier coefficients for congruently grown lithium niobate and 5 mol. \% magnesium oxide-doped lithium niobate, J. Opt. Soc. Am. B 14 3319-3322 (1997).

[23] L.E. Myers, R.C. Eckardt, M.M. Fejer, R.L. Byer, and W.R. Bosenberg, Multigrating quasi-phase-matched optical parametric oscillator in periodically poled LiNb) ${ }_{3}$, Opt. Lett. 21 591-593 (1996).

[24] A.L. Shawlow and C.H. Townes, Infrared and optical masers, Phys. Rev. 112, 1940-1949 (1958); 
Table 1. Signal Coherence Gain versus Incoherent Pump Bandwidth

\begin{tabular}{c|c|c|c|c|c}
\hline \hline case & $\Delta \nu_{p}(\mathrm{GHz})$ & $\Delta \nu_{s}(\mathrm{FSR})$ & $\Delta \nu_{s} / \Delta \nu_{p}$ & $\left\langle\Delta I_{s}\right\rangle /\left\langle\Delta I_{p}\right\rangle$ & $\tau_{c}^{\text {eff }} / \tau_{0}$ \\
\hline (a.) & 140.36 & $<1 / 512$ & $<1.26 \times 10^{-5}$ & $2.8 \times 10^{-3}$ & 0.60 \\
(b.) & 88.49 & $\sim 4$ & 0.041 & $3.4 \times 10^{-3}$ & 0.95 \\
(c.) & 67.69 & $\sim 4$ & 0.053 & $3.6 \times 10^{-3}$ & 1.24 \\
(d.) & 56.59 & $<1 / 512$ & $<3.14 \times 10^{-5}$ & $3.3 \times 10^{-3}$ & 1.49 \\
(e.) & 49.71 & $<1 / 512$ & $<3.57 \times 10^{-5}$ & $3.6 \times 10^{-3}$ & 1.70 \\
(f.) & 34.59 & $<1 / 512$ & $<5.13 \times 10^{-5}$ & $3.5 \times 10^{-3}$ & 2.44 \\
(g.) & 19.48 & $<1 / 512$ & $<9.12 \times 10^{-5}$ & $3.7 \times 10^{-3}$ & 4.34 \\
(h.) & 2.11 & $<1 / 512$ & $<8.40 \times 10^{-4}$ & $1.8 \times 10^{-3}$ & 40.0 \\
\hline \hline
\end{tabular}

$P_{p}=1520 \mathrm{~mW} ; \quad \tau_{0}=0.167 \mathrm{~ns} ; \quad L=3.5 L_{n l}=3.5 v_{p} \tau_{0}=8.00 \mathrm{~cm} ; \quad$ FSR $=0.910 \mathrm{GHz}$. 


\section{Figure captions}

Fig. 1 Group velocity dispersion for $\mathrm{Ti}^{2} \mathrm{LiNbO}_{3}$ at $20^{\circ} \mathrm{C}$ : experimental waveguide dispersion relation. The three marked values correspond to the point of operation of the nondegenerate QPM three-wave interaction in the singly resonant type I $\{e e e\}$ IOPO configuration (SR-IOPO).

Fig. 2 SR-IOPO for a signal intensity reflectivity $R=\rho_{s}^{2}=98.01 \%$, a Fabry-Perot cavity length $L=8.00 \mathrm{~cm}$ and an intensity signal attenuation $\alpha_{s}=0.06 \mathrm{~cm}^{-1}$ : Signal and idler output power (in log units) as function of the input pump power of spectral width $\Delta \nu_{p}=86.1 \mathrm{GHz}$ which coincide with the characteristics for a narrow coherent pump (plotted in the idler curve by the + symbols for the coherent pump, and by the * symbols for the incoherent pump).

Fig. 3 SR-IOPO for a signal intensity reflectivity $R=\rho_{s}^{2}=98.01 \%$ : Spatial amplitude evolution in the cavity of length $L=3.24 L_{n l}=8.00 \mathrm{~cm}$ at time $t=16384 t_{r}=18 \mu \mathrm{s}$. The incoherent random phase modulated pump at the input $(\mathrm{z}=0)$ generates strong amplitude fluctuations during propagation to the output due to dispersion in the waveguide (upper graph). It transfers its incoherence to the growing (non-resonant) idler wave, moving at the same group velocity, whereas the resonant signal wave becomes highly coherent. The relative pump and idler fluctuation levels become almost the same as Fig. $4(\mathrm{a})$ and $4(\mathrm{~b})$.

Fig. 4 SR-IOPO for a signal intensity reflectivity $R=\rho_{s}^{2}=98.01 \%$ at time $t=$ $16384 t_{r}=18 \mu \mathrm{s}:$ (a) Temporal pump evolution with a relative average fluctuating amplitude $\left\langle\Delta\left|A_{p}(L, t)\right|\right\rangle=0.2349$ around the average output amplitude $\left\langle\left|A_{p}(L, t)\right|\right\rangle=$ 0.8297. (b) Temporal idler evolution with almost the same relative average fluctuating amplitude $\left\langle\Delta\left|A_{i}(L, t)\right|\right\rangle=0.2356$ as the pump, around the average output amplitude $\left\langle\left|A_{i}(L, t)\right|\right\rangle=0.1825$. (c) Temporal signal evolution with a strongly reduced relative average fluctuating amplitude $\left\langle\Delta\left|A_{s}(L, t)\right|\right\rangle=0.01708$ around the average output amplitude 
$\left\langle\left|A_{s}(L, t)\right|\right\rangle=0.1198$

Fig. 5 SR-IOPO for a signal intensity reflectivity $R=\rho_{s}^{2}=98.01 \%$ at time $t=16384 t_{r}=$ $18 \mu \mathrm{s}$ : (a) Incoherent pump power spectrum showing the broad-bandwidth $\Delta \nu_{p}=86.1$ $\mathrm{GHz}$ stochasticity. (b) Idler power spectrum showing the same broad-bandwidth stochasticity absorbed from the incoherent pump. (c) Coherent signal power spectrum (single line) resulting from the convection-induced phase-locking mechanism. (d) Coherent signal power spectrum in logarithmic scale. [In Figs.5(a)-5(c) the power densities are normalized to the maximum of the pump power density].

Fig. 6 SR-IOPO for a signal intensity reflectivity $R=\rho_{s}^{2}=98.01 \%$. Coherent signal power spectrum in $\log _{10}$ scale showing the attractor tendency to coherence with temporal evolution at times: (a) $t=4096 t_{r}=4.5 \mu \mathrm{s}$, (b) $t=8192 t_{r}=9 \mu \mathrm{s}$, (c) $t=12288 t_{r}=13.5 \mu \mathrm{s}$, and $(\mathrm{d}) t=16384 t_{r}=18 \mu \mathrm{s}\left(\right.$ where $t_{r}=2 n_{s} L / c=1.1 \mathrm{~ns}$ is the signal round-trip time). The signal wave attains its asymptotics and emerges from the cavity with a bandwidth $\Delta \nu_{s}<2.0 \times 10^{-5} \Delta \nu_{p}$.

Fig. 7 SR-IOPO for a signal intensity reflectivity $R=\rho_{s}^{2}=98.01 \%$ at time $t=$ $16384 t_{r}=18 \mu \mathrm{s}$ [case (c.) of Table 1]: (a) Incoherent pump power spectrum of bandwidth $\Delta \nu_{p}=67.69 \mathrm{GHz}$. (b) Idler power spectrum showing the broad-bandwidth stochasticity absorbed from the incoherent pump. (c) Multiline signal power spectrum resulting from a non efficient coherence transfer. (d) Signal power spectrum in logarithmic scale. [In Figs.7(a)-7(c) the power densities are normalized to the maximum of the pump power density]. 


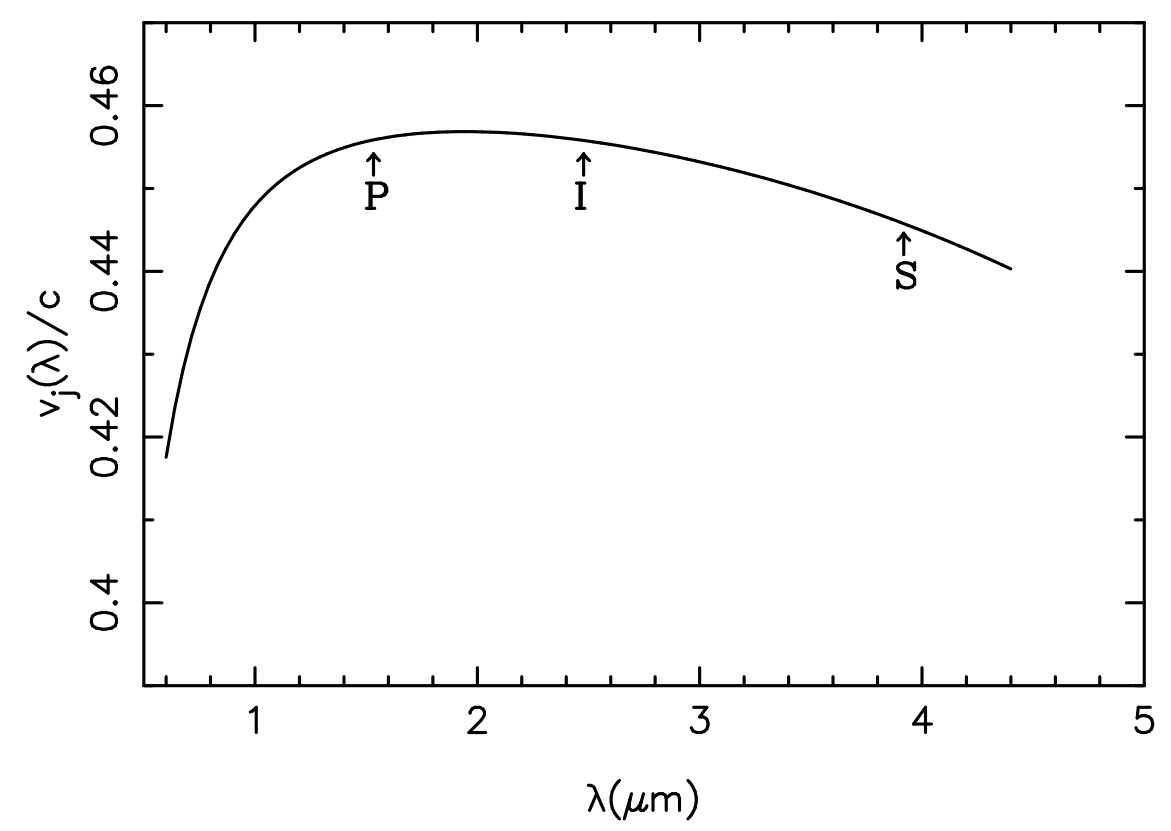

Figure 1: 


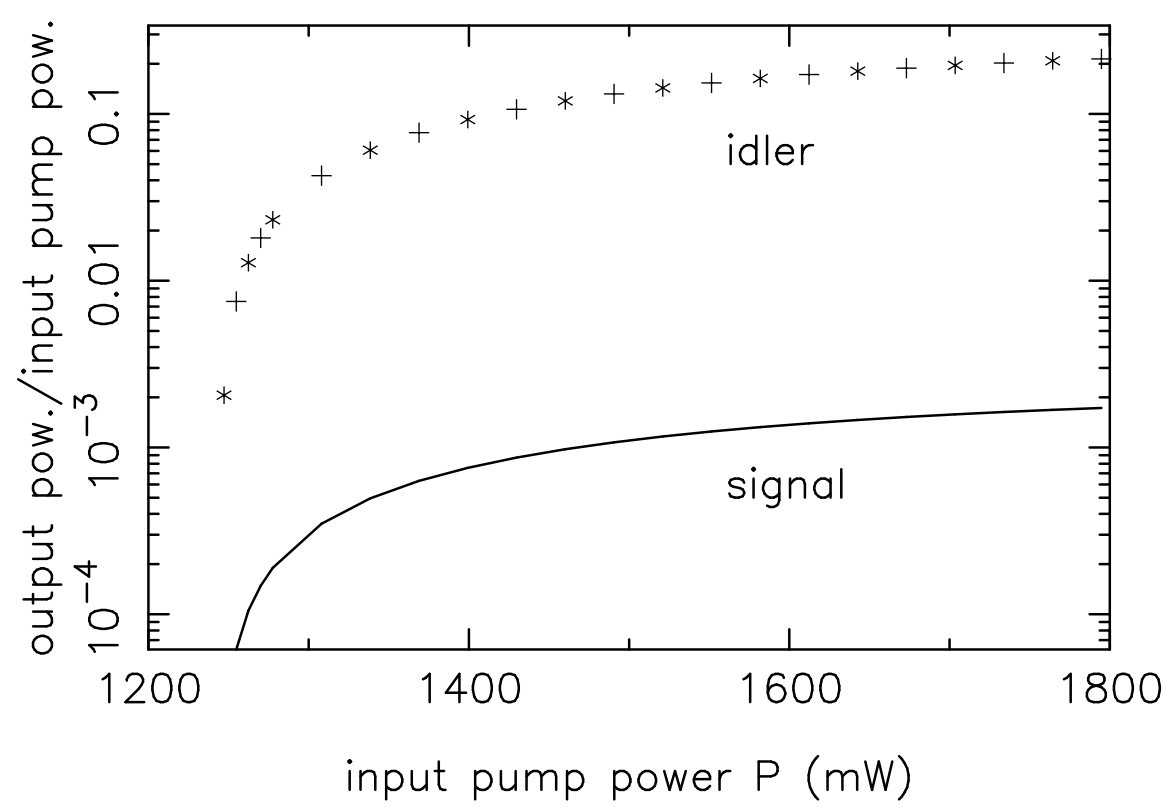

Figure 2:

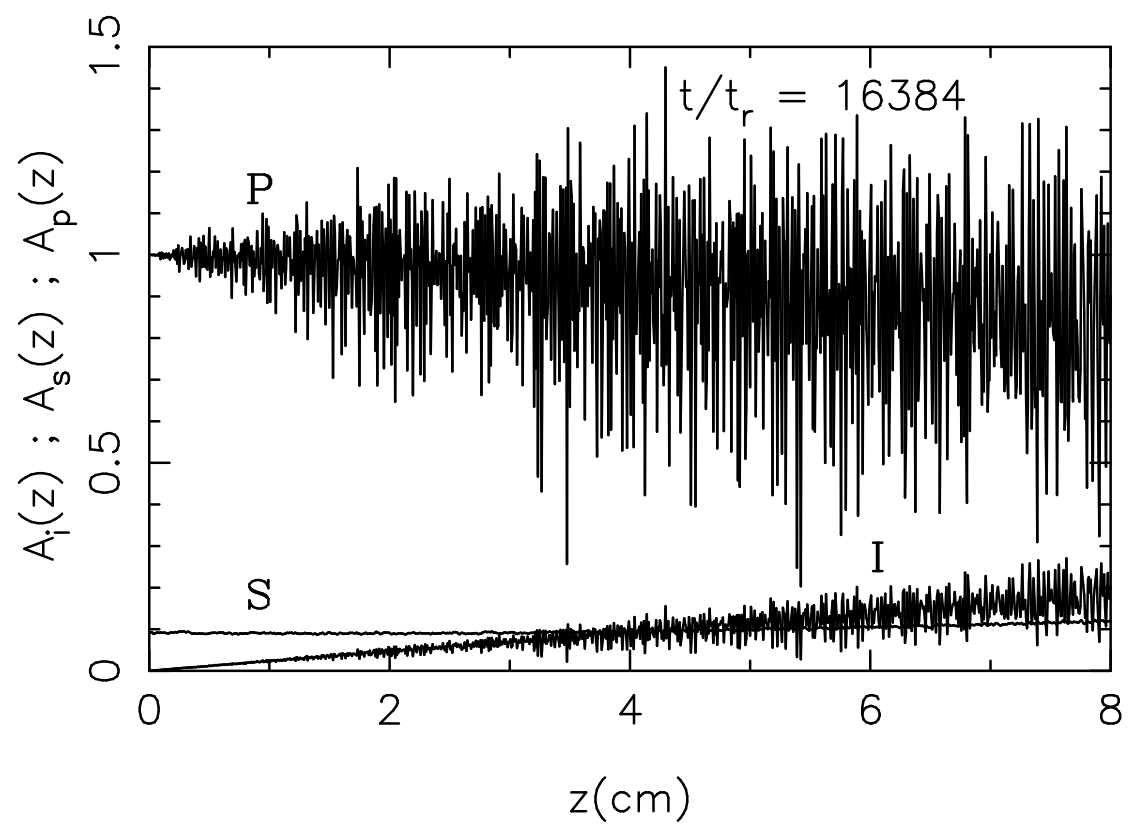

Figure 3: 

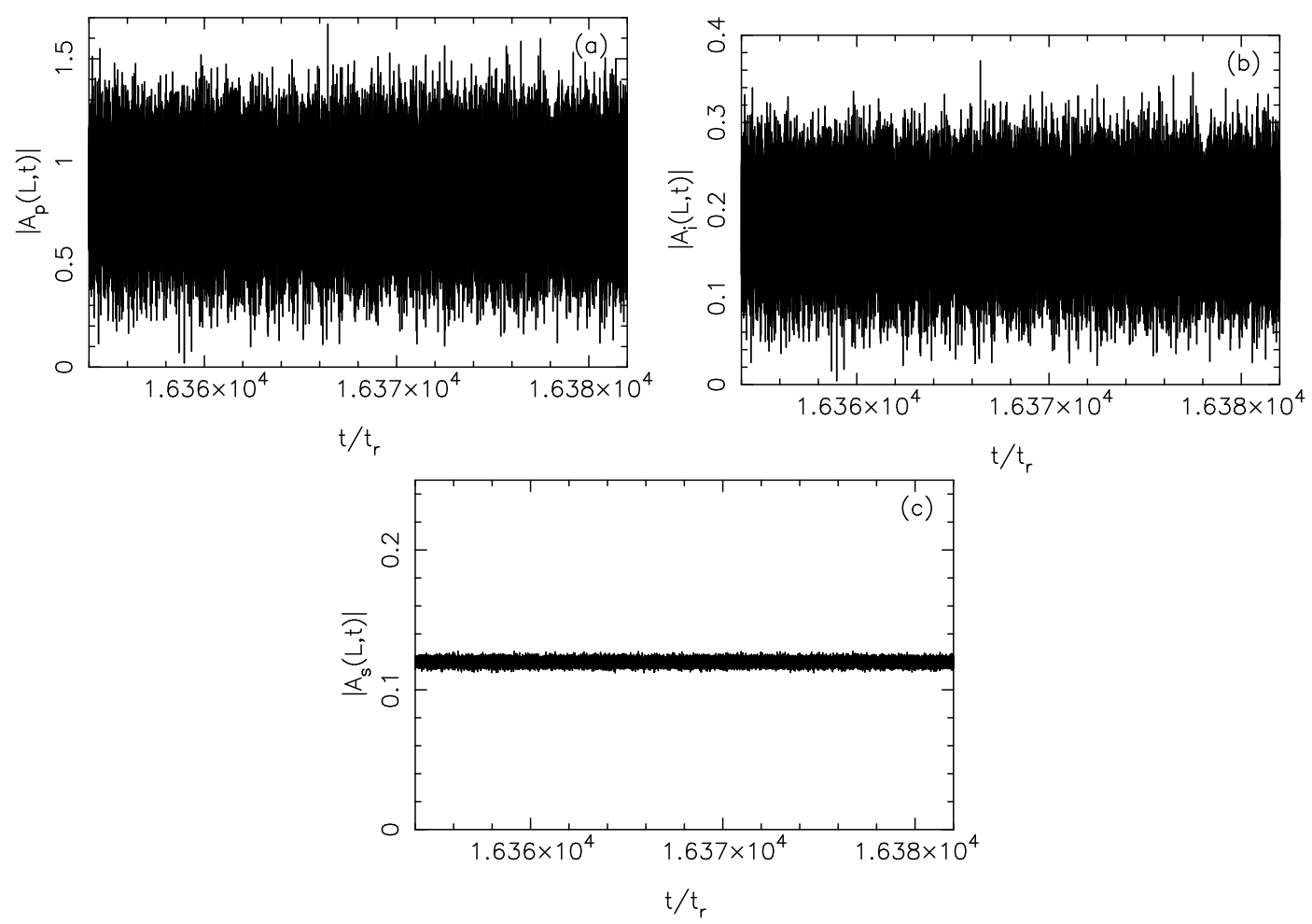

Figure 4:
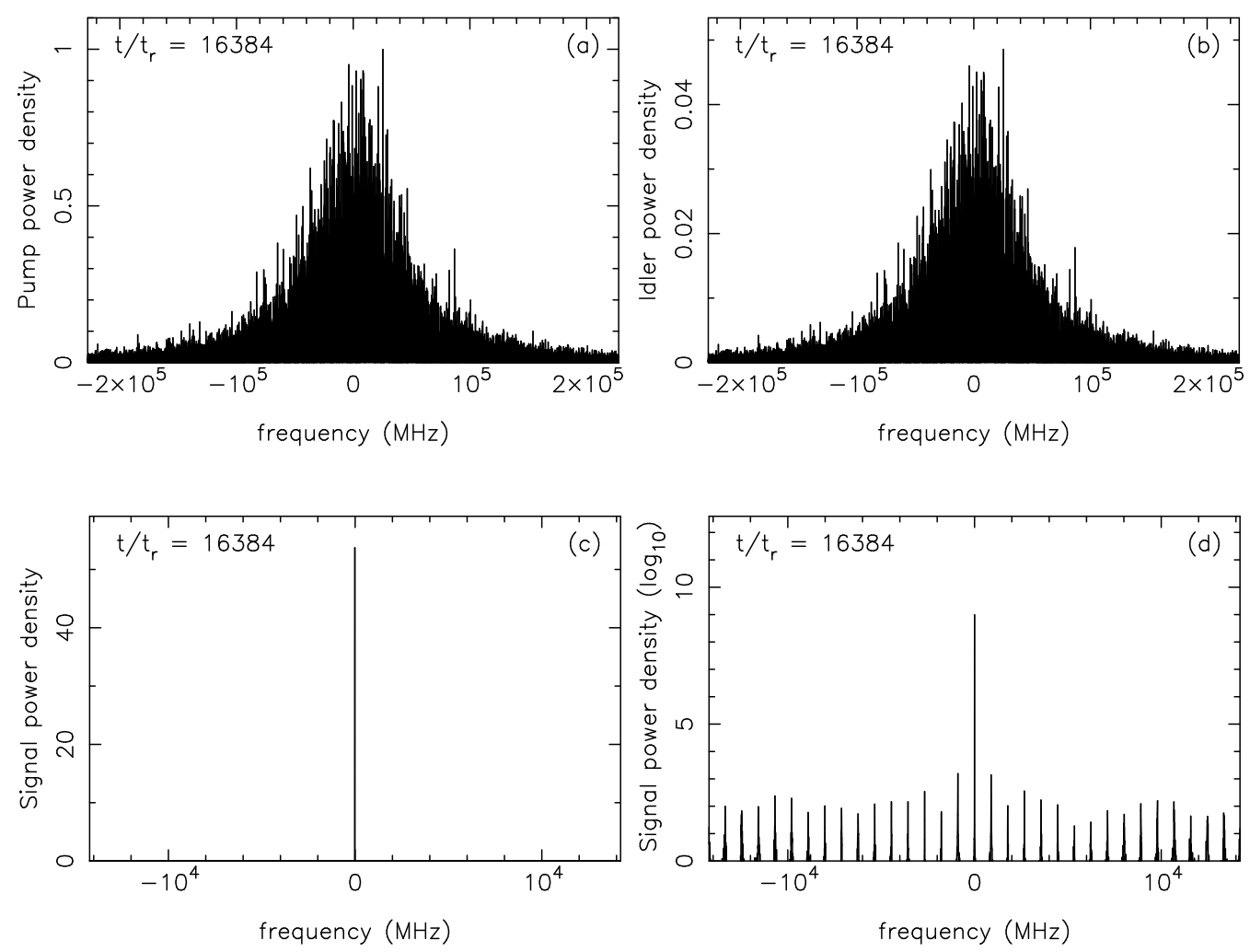

Figure 5: 

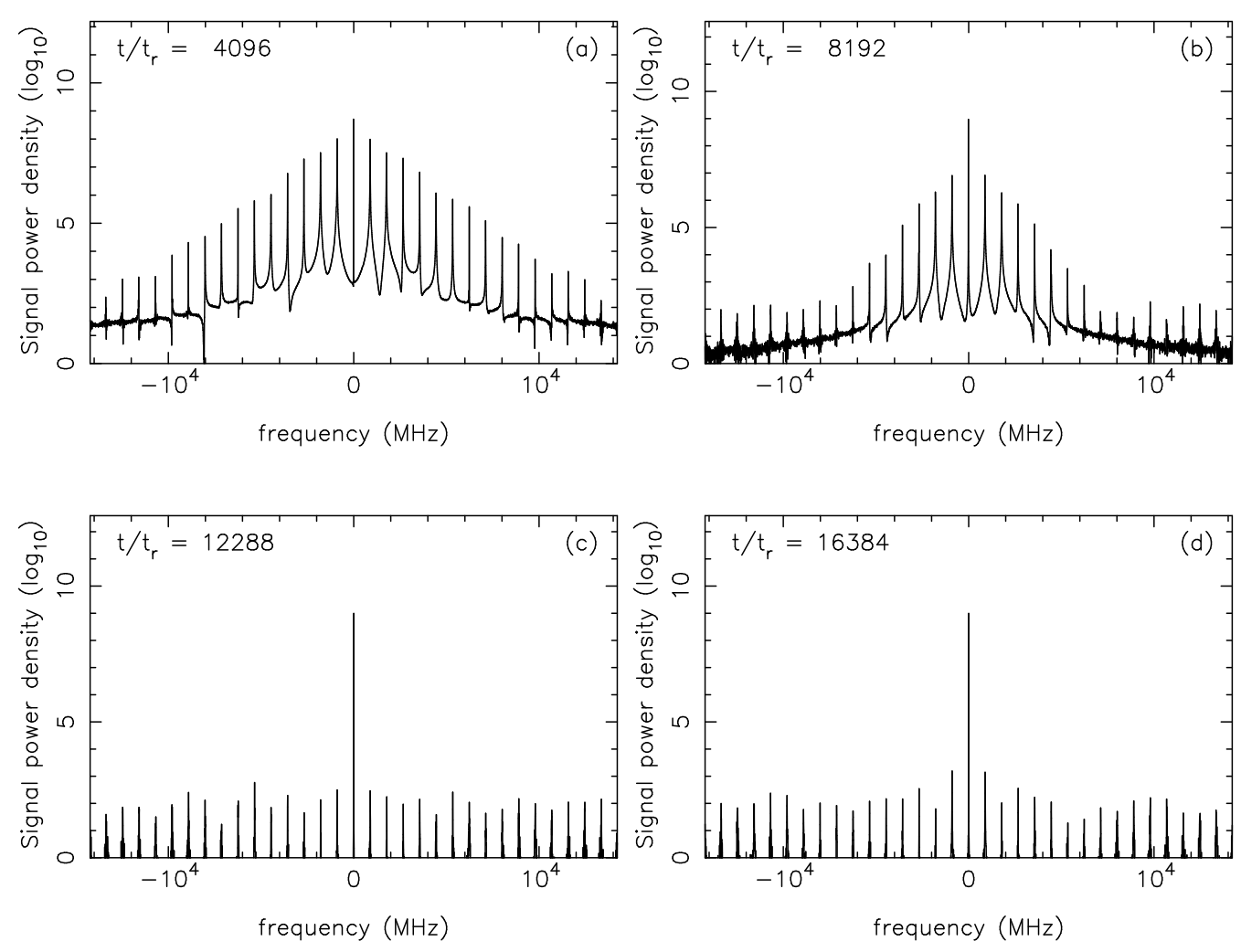

Figure 6:
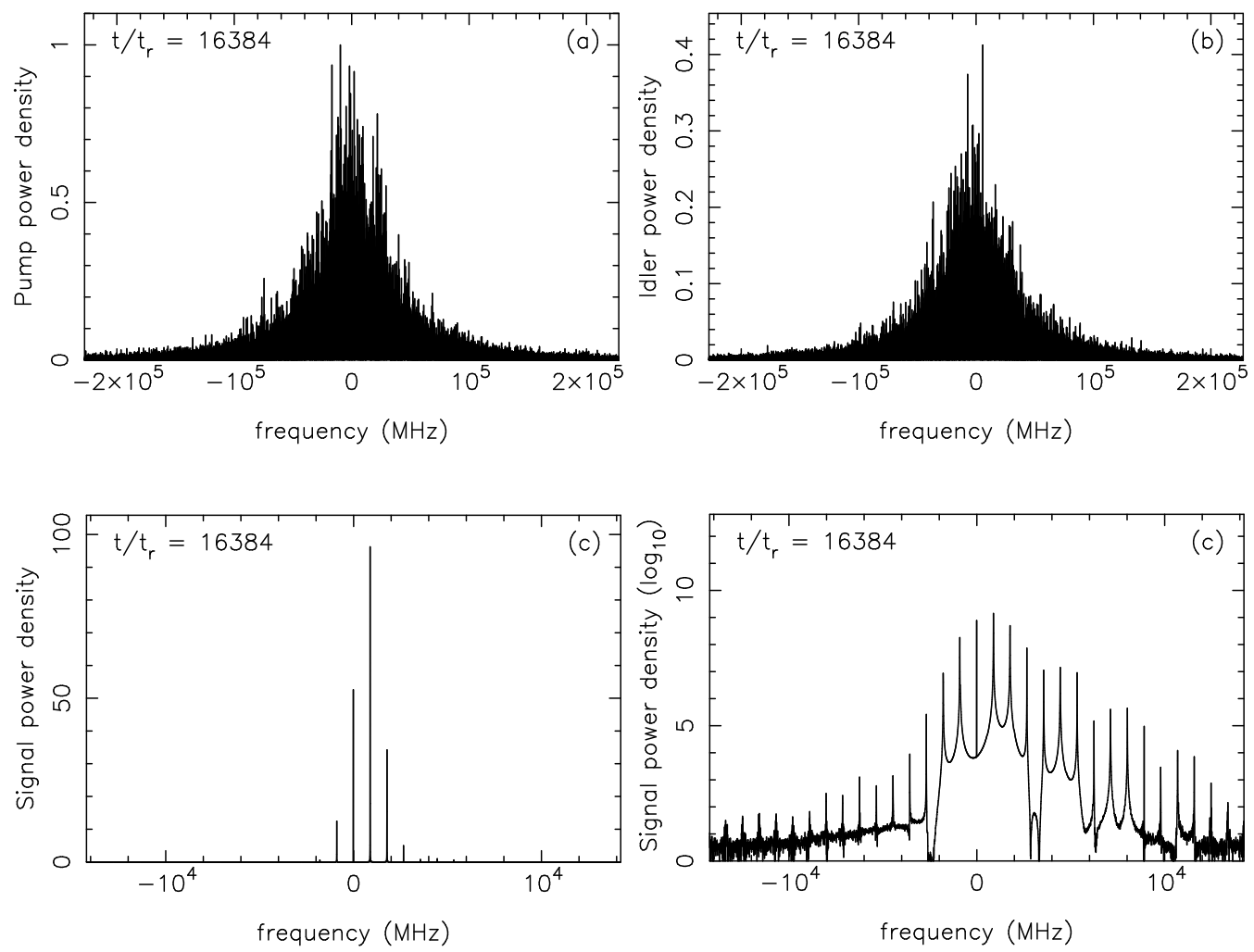

Figure 7: 